\title{
Temporal morphological changes in the Imhotep region of comet 67P/Churyumov-Gerasimenko
}

\author{
O. Groussin ${ }^{1}$, H. Sierks ${ }^{2}$, C. Barbieri ${ }^{3}$, P. Lamy ${ }^{1}$, R. Rodrigo ${ }^{4,5}$, D. Koschny ${ }^{6}$, H. Rickman ${ }^{7,8}$, H. U. Keller ${ }^{9}, 10$, \\ M. F. A'Hearn ${ }^{11}$, A.-T. Auger ${ }^{1,12}$, M. A. Barucci ${ }^{13}$, J.-L. Bertaux ${ }^{14}$, I. Bertini ${ }^{15}$, S. Besse ${ }^{6}$, G. Cremonese ${ }^{16}$, \\ V. Da Deppo ${ }^{17}$, B. Davidsson ${ }^{7}$, S. Debei ${ }^{16}$, M. De Cecco ${ }^{18}$, M. R. El-Maarry ${ }^{19}$, S. Fornasier ${ }^{13}$, M. Fulle ${ }^{20}$, \\ P. J. Gutiérrez ${ }^{21}$, C. Güttler ${ }^{2}$, S. Hviid ${ }^{3}$, W.-H Ip ${ }^{22}$, L. Jorda ${ }^{1}$, J. Knollenberg ${ }^{3}$, G. Kovacs ${ }^{2}$, J. R. Kramm², E. Kührt ${ }^{3}$, \\ M. Küppers ${ }^{23}$, L. M. Lara ${ }^{21}$, M. Lazzarin ${ }^{3}$, J. J. Lopez Moreno ${ }^{21}$, S. Lowry ${ }^{24}$, S. Marchi ${ }^{25}$, F. Marzari ${ }^{3}$, \\ M. Massironi ${ }^{15,26}$, S. Mottola ${ }^{3}$, G. Naletto ${ }^{15,17,27}$, N. Oklay ${ }^{2}$, M. Pajola ${ }^{15}$, A. Pommerol ${ }^{19}$, N. Thomas ${ }^{19}$, I. Toth ${ }^{28}$, \\ C. Tubiana ${ }^{2}$, and J.-B. Vincent ${ }^{2}$ \\ (Affiliations can be found after the references)
}

Received 21 July 2015 / Accepted 8 September 2015

\begin{abstract}
Aims. We report on the first major temporal morphological changes observed on the surface of the nucleus of comet 67P/Churyumov-Gerasimenko in the smooth terrains of the Imhotep region.

Methods. We used images of the OSIRIS cameras onboard Rosetta to follow the temporal changes from 24 May 2015 to 11 July 2015.

Results. The morphological changes observed on the surface are visible in the form of roundish features that are growing in size from a given location in a preferential direction at a rate of 5.6-8.1 $\times 10^{-5} \mathrm{~m} \mathrm{~s}^{-1}$ during the observational period. The location where the changes started and the contours of the expanding features are bluer than the surroundings, which suggests that ices $\left(\mathrm{H}_{2} \mathrm{O}\right.$ and/or $\left.\mathrm{CO}_{2}\right)$ are exposed on the surface. However, sublimation of ices alone is not sufficient to explain the observed expanding features. No significant variations in the dust activity pattern are observed during the period of changes.
\end{abstract}

Key words. comets: individual: 67P/Churyumov-Gerasimenko - comets: general - methods: data analysis

\section{Introduction}

Comets are among the most primitive bodies of our solar system and contain clues to constrain its formation and evolution (e.g., Weidenschilling 2004). They are active bodies that eject gas and dust into space during their orbit around the Sun. A key scientific question for understanding how comets work and whether they still contain pristine materials at or near their surface is how the nucleus changes with time and to which extent activity modifies its surface. The Rosetta spacecraft, which has been orbiting comet 67P/Churyumov-Gerasimenko (67P) since August 2014, offers a unique opportunity to tackle this fundamental question.

The only changes observed so far on the surface of a comet nucleus are those of 9P/Tempel 1, which was visited twice: in 2005 by the Deep Impact spacecraft (A'Hearn et al. 2005) and in 2011 by the Stardust spacecraft (Veverka et al. 2013). The morphological changes in this comet are restricted to a small area located near the largest smooth terrain. The two main detected changes are a retreat of up to $50 \mathrm{~m}$ of the boundaries of the smooth flow in at least two places and the merging of three roundish depressions into a larger one (Veverka et al. 2013).

Concerning the nucleus of 67P, modeling by Keller et al. (2015) predicts that it may locally lose up to $3.5-14.5 \mathrm{~m}$ per perihelion passage, assuming a dust-to-gas ratio of 4 (Rotundi et al. 2015). The erosion is nonuniform across the surface and strongly connected to insolation, with the strongest erosion in the southern hemisphere, which is the most illuminated hemisphere at perihelion. Observational evidence of past changes is also found on the nucleus surface, in the form of mass wasting (Sierks et al. 2015; Thomas et al. 2015b) and material transport (Thomas et al. 2015a). Since these changes precede the Rosetta rendezvous, it is not possible to know their timescale, and in particular, whether they result from a single or from multiple perihelion passages.

Although 67P had already lost $20 \%$ of its total mass loss per perihelion passage (Keller et al. 2015) until 24 May 2015, the OSIRIS cameras (Keller et al. 2007) onboard Rosetta had only detected subtle changes on the surface (e.g., in the Hapi region), whose authenticity is still being evaluated. In this article, we report on the first major temporal changes observed on the surface of $67 \mathrm{P}$ in the smooth terrains of the Imhotep region. This region, which presents a wide variety of terrains and morphologies, is a good candidate for being an active region at perihelion (Auger et al. 2015; Keller et al. 2015; Vincent et al. 2013). The most remarkable features are the smooth terrains, which extend over $0.8 \mathrm{~km}^{2}$ for the largest one, and the roundish features observed near the gravitational low of the region that have been interpreted as ancient degassing conduits by Auger et al. (2015).

\section{Observations}

\subsection{Temporal evolution}

Figure 1 shows the temporal variations of the Imhotep region from 24 May 2015 to 11 July 2015. Since the Rosetta arrival around 67P in August 2014 until 24 May 2015, no changes 


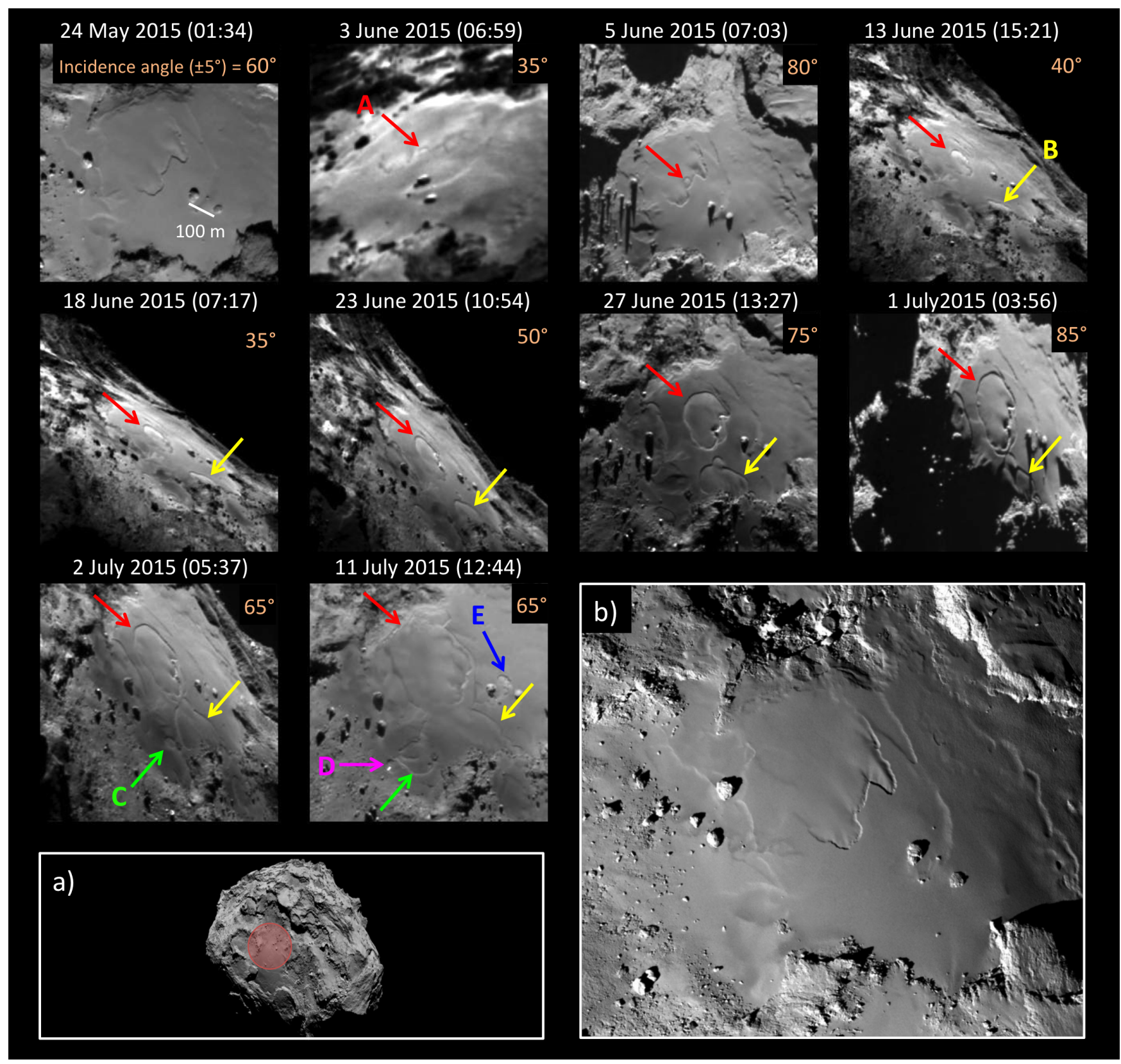

Fig. 1. Temporal evolution of the smooth terrains of Imhotep from 24 May 2015 to 11 July 2015 (UT). The arrows indicate the position of the five main detected changes, labeled A to E. The spatial resolution improved from $4.3 \mathrm{~m} /$ pix to $3.0 \mathrm{~m} / \mathrm{pix}$ over the period. The white line on $24 \mathrm{May}$ shows a $100 \mathrm{~m}$ reference scale that corresponds to the distance between two remarkable boulders that are visible in all images. Panel a) shows the regional context with the region of changes highlighted in red. Panel b) shows the smooth terrains at high spatial resolution (1 m/pix) in September 2014, before the changes (adapted from Auger et al. 2015). All the images were acquired with the Narrow Angle Camera (NAC) of the OSIRIS instrument.

had been detected in this region, down to the decimeter scale. Starting 3 June 2015, a first roundish feature (A) appeared on the surface and expanded in the following days. On 13 June 2015, a second roundish feature (B) appeared and also expanded. On 2 July 2015, a third feature (C) appeared and expanded. On 11 July 2015, the two additional features (D and E) appeared. At the end of the observational period, features A, B, and $\mathrm{C}$ merged and feature $\mathrm{A}$ reached the edges of the smooth terrains.

Each feature is growing in size from a given location in a preferential direction in a roughly circular pattern. Features originate from scarps (A), cliffs (B), terrain discontinuities $(\mathrm{C}$ and $\mathrm{E})$, or edges (D). The expansion rate of the two main features $A$ and $B$ is $8.1 \pm 0.8 \times 10^{-5} \mathrm{~m} \mathrm{~s}^{-1}$ and $5.6 \pm 0.6 \times 10^{-5} \mathrm{~m} \mathrm{~s}^{-1}$ respectively, this rate is constant from observation to observation. On 2 July 2015, the diameter reached $\sim 220 \mathrm{~m}$ for feature A and $\sim 140 \mathrm{~m}$ for feature $\mathrm{B}$, with rims of $5 \pm 2 \mathrm{~m}$. In one month, these dramatic morphological changes have already modified more than $40 \%$ of the surface of the largest smooth terrain of Imhotep, and they appear to be still ongoing.

\subsection{Bluish materials appear on the surface}

Figure 2 shows the blue-to-orange or blue-to-red color ratios of the evolving regions. The starting terrain of features $\mathrm{A}, \mathrm{D}$, and $\mathrm{E}$ is bluer than the surroundings and also brighter (best viewed on 11 July). The contours of the expanding features are also slightly 


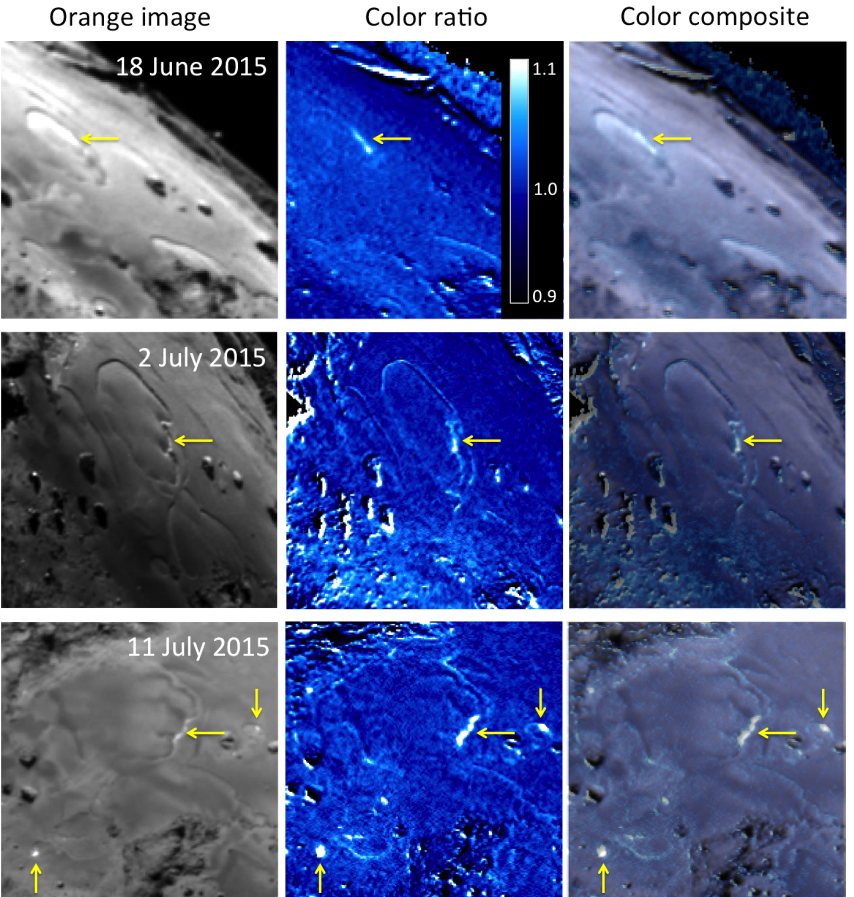

Fig. 2. Blue $(481 \mathrm{~nm})$ over orange $(649 \mathrm{~nm})$ or red $(701 \mathrm{~nm})$ color ratios of the evolving region on 18 June 2015 (upper row), 2 July 2015 (middle row), and 11 July 2015 (lower row). The first column shows a context image of the region, the second column shows the color ratio (blue-toorange for 18 June and 2 July, blue-to-red for 11 July), and the third column shows a color-composite image made of the first two columns. The yellow arrows indicate where features A, D, and E started (Fig. 1), with a material bluer than average (value above 1 in the color ratio). The images were acquired with the NAC.

bluer. The spectrophotometric analysis reveals that the bluest terrains are almost neutral in the range $500-950 \mathrm{~nm}$, whereas the spectral slope of the average terrain is $16 \%$ per $100 \mathrm{~nm}$ in the same wavelength range, that is, redder than the Sun.

This bluer material strongly suggests that ice is exposed on the surface (Pommerol et al. 2015; Fornasier et al. 2015; Capaccioni et al. 2015). This fresh material was probably buried below a dust deposit that has been removed by the erosion processes responsible for the observed changes. The presence of material enriched in volatiles in the first meters below the surface is supported by the low thermal inertia of $10-50 \mathrm{~J} \mathrm{~K}^{-1} \mathrm{~m}^{-2} \mathrm{~s}^{-0.5}$ of the nucleus (Gulkis et al. 2015) and by modeling (e.g., Prialnik et al. 2004), which both show that only the top few meters are affected by insolation. Since $\mathrm{H}_{2} \mathrm{O}$ and $\mathrm{CO}_{2}$ where detected in the coma above Imhotep (Hässig et al. 2015), the ice exposed on the surface might be composed of $\mathrm{H}_{2} \mathrm{O}$ and/or $\mathrm{CO}_{2}$.

\subsection{Link with dust activity}

We monitored the dust activity in the Imhotep region before and during the period of changes (Fig. 3). We did not detect any significant variations in the dust activity pattern above this region, and in particular, no increase in the number and intensity of dust jets above features A and B. More precisely, whereas several narrow collimated jets might originate from the smooth region, no strong jets are associated specifically with features A or B.

Although no significant variations are observed in the dust activity, we cannot exclude an increase in the gas production rate $\left(\mathrm{H}_{2} \mathrm{O}, \mathrm{CO}_{2}\right.$, or $\left.\mathrm{CO}\right)$ during the observational period, unfortunately beyond the capabilities of the OSIRIS instrument.
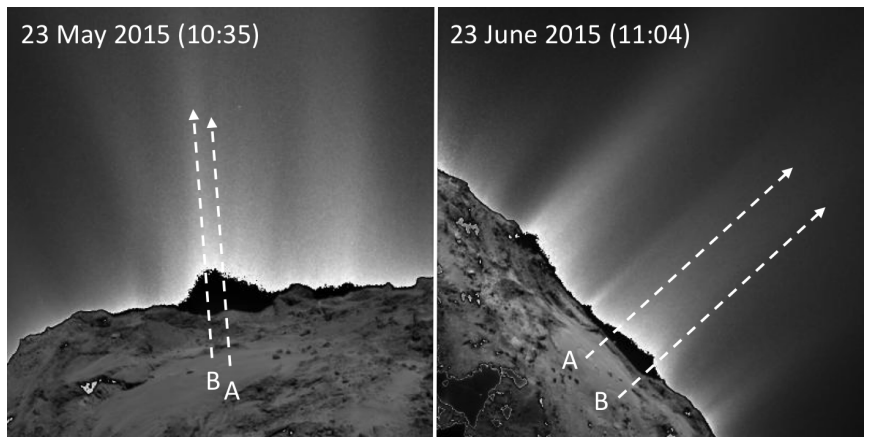

Fig. 3. Activity above Imhotep on 23 May 2015 and 23 June 2015 (UT), before and after the changes started. The local time at the nucleus differs by less than $30 \mathrm{~min}$ between the two images. The white arrows indicate the position where we would expect an increase in activity if jets were emitted from features A or B. The images were acquired with the NAC, more than $50^{\circ}$ away from nadir.

As an example, the strongest water and dust jets on comet 103P/Hartley 2 did not originate from the same region; water originated in the neck and the dust in the small lobe (A'Hearn et al. 2011).

\section{Discussions and conclusions}

We observe a collapse of a considerable thickness $(\sim 5 \mathrm{~m})$ of the upper surface, which is occurring in an organized way and propagates across the surface. This process starts from scarps, cliffs, terrain discontinuities, or edges, where the dust deposit is thinner and where the seasonal heat wave first reaches the underlying volatile-rich materials. In May-July 2015, these terrains were oriented toward sunrise; they are strongly illuminated in the morning, and the Sun is at its zenith above them, which means that they are probably local spots that are hotter than the neighborhood. The expansion moves preferentially away from these scarps or cliffs, which are consolidated materials and hence harder to erode. Later on, the expansion may pass this natural barrier, as it was the case for feature A on 27 June (Fig. 1). Each feature continues to grow in size in the sunrise direction, until it reaches another feature or the edges of the smooth terrain, and then it stops.

We calculated that the erosion rate for $\mathrm{H}_{2} \mathrm{O}$ and $\mathrm{CO}_{2}$ ice exposed on the surface can reach $4.2 \times 10^{-7} \mathrm{~m} \mathrm{~s}^{-1}$ and $2.8 \times 10^{-6} \mathrm{~m} \mathrm{~s}^{-1}$, respectively, at 1.4 au (heliocentric distance of $67 \mathrm{P}$ in June 2015), assuming a density of $535 \mathrm{~kg} \mathrm{~m}^{-3}$ (Preusker et al. 2015). This is much slower than the observed lateral expansion rate of 5.6-8.1 $\times 10^{-5} \mathrm{~m} \mathrm{~s}^{-1}$, which means that the sublimation of ices alone is not sufficient to explain the observed expanding features. Although it remains speculation at this stage, the eroding process might be exacerbated by the low tensile strength of tens of $\mathrm{Pa}$ of the cometary material (Groussin et al. 2015), which can easily erode in large chunks of several meters (Pajola et al. 2015), or the erosion might be driven by additional energetic processes such as clathrate destabilization or amorphous water ice crystallization (Mousis et al. 2015).

The lack of enhanced dust activity in the coma during the period of changes suggests that the dust deposit is dominated by millimeter-sized or larger particles and not by microscopic ones. Grains up to the decimeter scale are observed in the smooth terrains of Imhotep (Auger et al. 2015). During the eroding process, a fraction of dust particles falls and accumulates at the foot of the expanding features, or particles are transported slightly farther away, toward the center of the newly formed roundish features. This material transport might explain the bumpy shape 
of feature A (1 July, Fig. 1), with more material in the center than on the edges. The fraction of particles that escape the nucleus is unkown and cannot be determined by OSIRIS alone. We also add that if the nucleus is porous, a significant fraction of dust particles may sink into it during this process. Finally, it is worth mentioning that the surface material in the evolving regions looks photometrically essentially the same before and after the changes (Fig. 1).

If the same erosion process applies to the putative ancient degassing conduits of Imhotep (Auger et al. 2015), most of which are covered by a dust deposit, they might also be rejuvenated and become active at each perihelion passage. More generally, if activity increases significantly on Imhotep, it might be the source of the strong equatorial jet observed from Earth at the previous perihelion passage (Vincent et al. 2013). The hypothesis of such seasonal events is also supported by the fact that the height of the rims of the expanding features is a few meters, which corresponds to the depth to which the seasonal heat wave penetrates the nucleus (Gulkis et al. 2015).

The question is why such major and rapid changes on the surface are first and currently only observed in the smooth terrains of Imhotep. The northern hemisphere, which is also covered in several places by a dust deposit (El-Maarry et al. 2015), receives ten times less solar energy than Imhotep and is colder on average (Keller et al. 2015); if the same process takes place (e.g., in the Hapi region), it is on a longer timescale. The southern hemisphere is, on average, strongly eroded by several meters at each perihelion passage and therefore lacks dust deposits. Imhotep, which is located in a gravitational low able to retain and accumulate dust deposits, and where solar energy reaches $70-80 \%$ of the maximum energy received by the nucleus (Keller et al. 2015), is the proper location for such changes to take place.

It is therefore tempting to see similarities with the morphological changes observed on 9P between 2005 and 2011 (Sect. 1), which are also linked to the largest smooth terrain on the surface and occurred in a gravitational low like Imhotep (Thomas et al. 2007). The retreat of the scarp from the smooth flow is best interpreted by the progressive sublimation of material over several months (Veverka et al. 2013), that is, a slow process compared to what we see on Imhotep. However, the merging of multiple depressions might be another example of the type of fast-erosion process observed on Imhotep, with the strong limitation that we do not know the timescale of this event on 9P.

The dramatic changes observed on Imhotep are a spectacular event, unique to comets, with a currently unpredictable end state. We will continue to carefully monitor this region during the coming months to better constrain the erosion processes responsible for these changes. Registering changes on 67P remains a key scientific objective for all Rosetta instruments to better understand how comets work and evolve.

Acknowledgements. OSIRIS was built by a consortium of the Max-PlanckInstitut für Sonnensystemforschung, Göttingen, Germany; the CISAS University of Padova, Italy; the Laboratoire d'Astrophysique de Marseille, France; the Instituto de Astrofísica de Andalucia, CSIC, Granada, Spain; the Research and Scientific Support Department of the ESA, Noordwijk, Netherlands; the Instituto Nacional de Técnica Aeroespacial, Madrid, Spain; the Universidad Politéchnica de Madrid, Spain; the Department of Physics and Astronomy of Uppsala University, Sweden; and the Institut für Datentechnik und Kommunikationsnetze der Technischen Universität Braunschweig, Germany. The support of the national funding agencies of Germany (DLR), France (CNES), Italy (ASI), Spain (MEC), Sweden (SNSB), and the ESA Technical Directorate is gratefully acknowledged. We thank the Rosetta Science Operations Centre and the Rosetta Mission Operations Centre for the successful rendezvous with comet 67P/Churyumov-Gerasimenko.

\section{References}

A'Hearn, M. F., Belton, M. J. S., Delamere, W. A., et al. 2005, Science, 310, 258 A'Hearn, M. F., Belton, M. J. S., Delamere, W. A., et al. 2011, Science, 332, 1396

Auger, A.-T., Groussin, O., Jorda, L., et al. 2015, A\&A, 583, A35

Capaccioni, F., Filacchione, G., Erard, S., et al. 2015, EGU, 12375

El-Maarry, M. R., Thomas, N., Giacomini, L., et al. 2015, A\&A, 583, A26 Fornasier, S., Hasselmann, P. H., Barucci, M. A., et al. 2015, A\&A, 583, A30 Groussin, O., Jorda, L., Auger, A.-T., et al. 2015, A\&A, 583, A32

Gulkis, S., Allen, M., von Allmen, P., et al. 2015, Science, 347, 709

Hässig, M., Altwegg, K., Balsiger, H., et al. 2015, Science, 347, 0276

Keller, H. U., Barbieri, C., Lamy, P., et al. 2007, Space Sci. Rev., 128, 433

Keller, H. U., Mottola, S., Davidsson, B., et al. 2015, A\&A, 583, A34

Mousis, O., Guilbert-Lepoutre, A., Brugger, B., et al. 2015, ApJL, submitted

Pajola, M., Vincent, J.-B., Güttler, C., et al. 2015, A\&A, 583, A37

Pommerol, A., Thomas, N., El-Maarry, M. R., et al. 2015, A\&A, 583, A25

Preusker, F., Scholten, F., Matz, K.-D., et al. 2015, A\&A, 583, A33

Prialnik, D., Benkhoff, J., \& Podolak, M. 2004, Comets II (Tucson: University of Arizona Press), 359

Rotundi, A., Sierks, H., Della Corte, V., et al. 2015, Science, 347, 3905

Sierks, H., Barbieri, C., Lamy, P. L., et al. 2015, Science, 347, 1044

Thomas, P. C., Veverka, J., Belton, M. J., et al. 2007, Icarus, 187, 4

Thomas, N., Davidsson, B., El-Maarry, M. R., et al. 2015a, A\&A, 583, A17

Thomas, N., Sierks, H., Barbieri, C., et al. 2015b, Science, 347

Veverka, J., Klaasen, K., A'Hearn, M., et al. 2013, Icarus, 222, 424

Vincent, J.-B., Lara, L., Tozzi, G., Lin, Z., \& Sierks, H. 2013, A\&A, 549, A121 Weidenschilling, S. 2004, Comets II, 745, 97

1 Aix Marseille Université, CNRS, LAM (Laboratoire d'Astrophysique de Marseille) UMR 7326, 13388 Marseille, France

2 Max-Planck-Institut für Sonnensystemforschung, 37077 Göttingen, Germany

3 Department of Physics and Astronomy, Padova University, Vicolo dell'Osservatorio 3, 35122 Padova, Italy

4 Centro de Astrobiologia (INTA-CSIC), 28691 Villanueva de la Canada, Madrid, Spain

5 International Space Science Institute, Hallerstrasse 6, 3012 Bern, Switzerland

6 Scientific Support Office, European Space Agency, 2201 Noordwijk, The Netherlands

7 Department of Physics and Astronomy, Uppsala University, Box 516, 75120 Uppsala, Sweden

8 PAS Space Research Center, Bartycka 18A, 00716 Warszawa, Poland

9 Institute of Planetary Research, DLR, Rutherfordstrasse 2, 12489 Berlin Germany

10 Institute for Geophysics and Extraterrestrial Physics, TU Braunschweig, 38106, Germany

11 Department of Astronomy, University of Maryland, College Park, MD, 20742-2421, USA

12 Laboratoire GEOPS (Géosciences Paris Sud), Bat. 509, Université Paris Sud, 91405 Orsay Cedex, France

13 LESIA, Obs. de Paris, CNRS, Univ Paris 06, Univ. Paris-Diderot, 5 place J. Janssen, 92195 Meudon, France

14 LATMOS, CNRS/UVSQ/IPSL, 11 boulevard d'Alembert, 78280 Guyancourt, France

15 Centro di Ateneo di Studi ed Attività Spaziali, "Giuseppe Colombo" (CISAS), University of Padova, via Venezia 15, 35131 Padova, Italy

16 Department of Industrial Engineering, University of Padova, 35131 Padova, Italy

17 CNR-IFN UOS Padova LUXOR, via Trasea 7, 35131 Padova, Italy

18 UNITN, Universit di Trento, via Mesiano, 77, 38100 Trento, Italy

19 Physikalisches Institut, Sidlerstr. 5, University of Bern, 3012 Bern, Switzerland

20 INAF-Osservatorio Astronomico, via Tiepolo 11, 34143 Trieste, Italy

21 Instituto de Astrofisica de Andalucía (CSIC), Glorieta de la Astronomía s/n, 18008 Granada, Spain

22 Institute for Space Science, Nat. Central Univ., 300 Chung Da Rd., 32054 Chung-Li, Taiwan

23 Operations Department, European Space Astronomy Centre/ESA, PO Box 78, 28691 Villanueva de la Canada, Madrid, Spain

24 Centre for Astrophysics and Planetary Science, Schoold of Physical Sciences (SEPnet), The Univsersity of Kent, Canterbury, CT2 7NH, UK

25 Southwest Research Institute, 1050 Walnut St., Boulder, CO 80302, USA

26 INAF, Osservatorio Astronomico di Padova, 35122 Padova, Italy

27 University of Padova, Department of Information Engineering, via Gradenigo 6/B, 35131 Padova, Italy

28 Konkoly Observatory, 1525 Budapest, PO Box 67, Hungary 